\title{
THE HAHN-BANACH THEOREM AND THE LEAST UPPER BOUND PROPERTY
}

\author{
BY \\ R. J. SILVERMAN AND TI YEN
}

1. Introduction. Crucial to the proof of the Hahn-Banach theorem on the extension of linear functionals is the least upper bound property of the real number field. It is known that when the real number field is replaced by a boundedly complete vector lattice as the range space of the function, then Hahn-Banach extensions are possible $[2 ; 4]$. The question as to whether an ordered linear space which permits Hahn-Banach type extensions is a boundedly complete vector lattice is answered in this paper in the negative. However, when a minor additional necessary condition is imposed on the positive elements of the range space the Hahn-Banach extension property implies the least upper bound property.

2. Theorem. Let $V$ be a partially ordered linear space over the real number field $R$. That is, $V$ is a linear space with a transitive relationship ( $\geqq$ ) such that if $v_{1} \geqq v_{2}$, then $t v_{1} \geqq t v_{2}$ and $v_{1}+v \geqq v_{2}+v$ for every non-negative real number $t$ and all $v \in V$. The set $C=\{v \in V \mid v \geqq 0\}$ is the positive cone of $V$. The positive cone determines the ordering: $v_{1} \geqq v_{2}$ if and only if $v_{1}-v_{2} \in C$. Conversely, a nonempty set $C$ of elements in a vector space $V$ such that $x+y \in C$, $t x \in C$, for every $x, y \in C$ and non-negative real number $t$, is a positive cone relative to the ordering: $v_{1} \geqq v_{2}$ if and only if $v_{1}-v_{2} \in C$.

The ordered linear space $V$ with cone $C$ is lineally closed if every line, $l\left(v_{0}, v_{1}\right)=\left\{t v_{0}+(1-t) v_{1} \mid t \in R\right\}$ is either disjoint from $C$ or intersects $C$ in a closed segment. Equivalently, if $t v_{1} \geqq v$ for some $t \geqq 0$, where $v_{1} \geqq 0$, then $t_{v} v_{1} \geqq v$, where $t_{v}=$ inf $\left\{t \geqq 0 \mid t v_{1} \geqq v\right\}$.

The partially ordered linear space $V$ has the least upper bound property (LUBP) (i.e., $V$ is a boundedly complete vector lattice) if every set of elements with an upper bound has a least upper bound. If $V$ has the LUBP then of course every set of elements with a lower bound has a greatest lower bound. The least upper bound of a set is not necessarily unique unless it is assumed that $x \geqq y \geqq x$, implies $x=y$. In fact if $u$ is a least upper bound for a set $A$, then if $u \geqq u^{\prime} \geqq u, u^{\prime}$ is also a least upper bound for $A$.

The partially ordered linear space $V$ has the Hahn-Banach extension property (HBEP) if given (1) a real linear space $Y,(2)$ a linear subspace $X$ of $Y$, (3) a positive homogeneous subadditive function $p: Y \rightarrow V$, and (4) a distributive function $f: X \rightarrow V$ such that $f(x) \leqq p(x), x \in X$, then there is always a distributive extension $F: Y \rightarrow V$ of $f$ such that $F(y) \leqq p(y), y \in Y$.

It is proved in [2] that if $V$ has the LUBP then it has the HBEP. It will be proved here that if $V$ is lineally closed and has the HBEP, then $V$

Received by the editors November 10, 1956 and, in revised form, May 15, 1957. 
has the LUBP. That $V$ be lineally closed is a necessary condition is implied by the following theorem and the example presented at the end of the paper.

\section{TheOREM 1. If $V$ has the LUBP then $V$ is lineally closed.}

Proof. Consider $v_{1}>0$, v, and the set $\left\{t \geqq 0 \mid t v_{1} \geqq v\right\}$. Let $t_{v}=\inf \left\{t \geqq 0 \mid t v_{1} \geqq v\right\}$. One has $t_{v} v_{1} \geqq v$ if one shows that $t_{v} v_{1}$ is a greatest lower bound of $\left\{t v_{1} \mid t \geqq 0\right.$, $\left.t v_{1} \geqq v\right\}$. Let $w$ be a greatest lower bound of this set. Then $0 \leqq w-t_{v} v_{1} \leqq \epsilon v_{1}$ for every $\epsilon>0$. Hence $2\left(w-t_{v} v_{1}\right) \leqq \epsilon v_{1}$ for every $\epsilon>0$, since $w-t_{v} v_{1}$ is a greatest lower bound of $\left\{\epsilon v_{1} \mid \epsilon>0\right\}, 2\left(w-t_{v} v_{1}\right) \leqq w-t_{v} v_{1}$. Therefore, $t_{v} v_{1} \geqq w \geqq v$. Thus $V$ is lineally closed.

To begin the proof of the main theorem, one assumes that the positive cone $C$ of $V$ is reproducing, i.e., every element of $V$ is a difference of two elements in $C$. A cone $C$ is minihedral if every pair of elements in $C$ has a least upper bound. If $C$ is reproducing then every pair of elements has a least upper bound. Indeed, let $x=u-v, y=u^{\prime}-v^{\prime}$, where $u, u^{\prime}, v, v^{\prime} \in C$. Then $\sup (x, y)=\sup \left(x+v+v^{\prime}, y+v+v^{\prime}\right)-v-v^{\prime}$.

Lemma 1. Let $V$ be lineally closed and have the HBEP. Then $C$ is minihedral. $\left({ }^{1}\right)$

Proof. Let $v_{1}, v_{2} \in C$. Let $A$ be the subset of $V$ consisting of the elements $v$ such that $v_{1}, v_{2} \leqq v \leqq w$ (e.g., $w=v_{1}+v_{2}$ ). Let $X$ be the subspace of $V$ spanned by $A, v_{1}$, and $v_{2}$, and let $Y$ be the space obtained by adjoining to $X$ an ideal element $y_{0}$. Define a partial ordering in $Y$ by taking the cone generated by the positive cone of $X, A-y_{0}, y_{0}-v_{1}$, and $y_{0}-v_{2}$ to be the positive cone of $Y$.

If $x=a v+b v_{1}+c v_{2}(v \in A)$, then $x \leqq|a| v+|b| v_{1}+|c| v_{2} \leqq(|a|+|b|+|c|) w$, that is, for every $x \in X$, there exists $t \geqq 0$ such that $t w \geqq x$. If $y=a x+b y_{0}(x \in X)$, then $y \leqq a x+|b| y_{0} \leqq a x+|b| w$, for $y_{0} \geqq v_{1} \geqq 0$. Hence, for each $y \in Y$, there exists $t \geqq 0$ such that $y \leqq t w$. Let $t_{y}=\inf \{t \geqq 0 \mid t w \geqq y\}$, and define $p: Y \rightarrow V$ by $p(y)=t_{y} w$. It is easy to verify that $p$ is positive homogeneous and subadditive. Moreover, $p(x) \geqq x(x \in X)$, for $V$ is lineally closed. Therefore, since $V$ has HBEP, the identity map $f: X \rightarrow V$ has an extension $F: Y \rightarrow V$ such that $F(y) \leqq p(y), y \in Y$.

Now $F\left(y_{0}\right)-v_{i}=-F\left(v_{\imath}-y_{0}\right) \geqq-p\left(v_{i}-y_{0}\right)=-t_{v_{i}-y_{0}} w=0$, since $v_{i}-y_{0} \leqq 0$ and, consequently, $t_{v_{i}-y_{0}}=0(i=1,2)$. Thus $F\left(y_{0}\right)$ is an upper bound for $v_{1}$ and $v_{2}$ in $V$. Further, for every $v \in A, v-F\left(y_{0}\right)=-F\left(y_{0}-v\right) \geqq-p\left(y_{0}-v\right)$ $=-t_{y_{0}-v} w=0$, because $y_{0}-v \leqq 0$.

One shows that $F\left(y_{0}\right)$ is a least upper bound of $v_{1}$ and $v_{2}$. Suppose $v_{0} \geqq v_{1}, v_{2}$. In the above argument, if $v_{0}+F\left(y_{0}\right)$ is taken instead of $w$, a $v_{3}$ is obtained such that $v_{1}, v_{2} \leqq v_{3} \leqq v_{0}, F\left(y_{0}\right)$, since $v_{0}, F\left(y_{0}\right) \leqq v_{0}+F\left(y_{0}\right)$ belong to the new $A$. Since $v_{3} \leqq F\left(y_{0}\right), v_{3} \leqq w$, thus $v_{3} \in A$ and $v_{3} \geqq F\left(y_{0}\right)$. Consequently, $v_{0} \geqq F\left(y_{0}\right)$ and $F\left(y_{0}\right)$ is a least upper bound.

(1) The authors are indebted to Professor M. M. Day for pointing out a mistake in their original proof of this lemma. 
Lemma 2. Let $V$ be a lineally closed partially ordered linear space with reproducing cone $C$. If $V$ has the $H B E P$, then for any two nonempty subsets $A, B$ of $V$ such that $A \leqq B$ (i.e., $a \leqq b, a \in A, b \in B$ ), there is an element $c \in V$ such that $A \leqq c \leqq B$. That is, $V$ has the LUBP [2].

Proof. Assume that $B \geqq 0$. This can be done without loss of generality. For, if $a \leqq b$ then $a-a^{\prime} \leqq b-a^{\prime}$ for a fixed $a^{\prime} \in A$. Then the set $B-a^{\prime}$ consists of only positive elements. Further, $A \leqq c \leqq B$ if and only if $A-a^{\prime} \leqq c-a^{\prime}$ $\leqq B-a^{\prime}$.

Take $u_{0} \in B$ and $w_{0} \in A$. Let $A_{0}=\left\{v \mid v \in V, w_{0} \leqq v \leqq B\right\}$, and $B_{0}=\{v \mid v \in V$, $\left.A_{0} \leqq v \leqq u_{0}\right\}$. If there is a $v \in V$ such that $A_{0} \leqq v \leqq B_{0}$ then $A \leqq v \leqq B$. For if $w \in A$, then by Lemma $1 \sup \left(w, w_{0}\right)$ exists and is in $A_{0}$. Hence $v \geqq \sup \left(w, w_{0}\right)$ $\geqq w$. Similarly, if $u \in B$, then $\inf \left(u, u_{0}\right)$ exists and is in $B_{0}$ and $v \leqq \inf \left(u, u_{0}\right) \leqq u$.

Let $v_{0}=\sup \left(u_{0},-w_{0}\right)$. Let $X$ be the subspace spanned by $A_{0}, B_{0}$ and let $Y$ be the space obtained by adjoining to $X$ an ideal element $y_{0}$. Partially order $Y$ by taking the cone generated by the cone of $X, y_{0}-A_{0}$, and $B_{0}-y_{0}$ to be the positive cone.

For every element $x \in X, x=a u+b w, u \in B_{0}, w \in A_{0} . a u \leqq a u_{0}$ if $a \geqq 0$, or $a u \leqq-|a| w_{0}$ if $a \leqq 0$. Hence $a u \leqq|a| v_{0}$. Similarly, $b w \leqq|b| v_{0}$. Therefore, for each $x \in X, x \leqq t v_{0}$ for some $t \geqq 0$. If $y=x+a y_{0}(x \in X), y=x+a w_{0}+a\left(y_{0}-w_{0}\right)$ $\leqq x+a w_{0}+|a|\left(y_{0}-w_{0}\right) \leqq x+a w_{0}+|a|\left(u_{0}-w_{0}\right)$. Hence, for each $y \in Y$, there exists $t \geqq 0$ such that $t v_{0} \geqq y$. Define $p: Y \rightarrow V$ by $p(y)=t_{y} v_{0}$ where $t_{y}$ $=\inf \left\{t \geqq 0 \mid t v_{0} \geqq y\right\}$. Then $p(x) \geqq x(x \in X)$, because $V$ is lineally closed. Thus the identity map $f: X \rightarrow V$ can be extended to a distributive function $F: Y \rightarrow V$ such that $F(y) \leqq p(y), y \in Y$.

For $u \in B_{0}, u-F\left(y_{0}\right)=-F\left(y_{0}-u\right) \geqq-p\left(y_{0}-u\right)=0$, because $y_{0}-u \leqq 0$. Similarly, $F\left(y_{0}\right)-A_{0} \geqq 0$. Therefore, $A \leqq F\left(y_{0}\right) \leqq B$ and $V$ has the LUBP.

The following lemmas remove the assumption that the cone $C$ is reproducing.

Lemma 3. If $V$ is a partially ordered linear space and $A, B \subset V$ such that $A \leqq B, B \geqq 0$, then $A$ and $B$ are contained in $V_{1}$, the subspace spanned by the cone $C$ of $V$.

Proof. $A \subset B-(B-A) \subset V_{1}$.

Lemma 4. If $V$, a partially ordered linear space with cone $C$, has the HBEP, then the space $V_{1}$ spanned by $C$ also has the HBEP.

Proof. Consider a linear space $Y$, a subspace $X$ of $Y$, a positive homogeneous subadditive function $p: Y \rightarrow V_{1}$ and a distributive function $f: X \rightarrow V_{1}$ such that $f(x) \leqq p(x), x \in X$. Then since $V$ has the HBEP, there exists a distributive extension $F$ of $f$ from $Y$ to $V$ such that $F(y) \leqq p(y), y \in Y$. But then, $F(y)=p(y)-(p(y)-F(y))$ is in $V_{1}$.

Combining Lemmas $2-4$, one obtains: 
MAIN Theorem. If $V$ is a lineally closed ordered linear space with the $H B E P$, then $V$ has the LUBP.

3. Example. An example is presented to show that the HBEP does not by itself imply the LUBP for an ordered linear space $V$. Consider the two dimensional linear space $V$ of ordered pairs of real numbers with the following ordering: $(x, y) \geqq 0$ if and only if $y \geqq 0$ and if $y=0$, then $x=0$ (i.e., the cone is the open upper half plane plus the origin). The space $V$ does not have the LUBP. For consider the $x$-axis, the open upper half plane is its set of upper bounds. Neither is $V$ lineally closed, obviously.

The space $V$ does, however, have the HBEP. For consider $Y, X, p, f$, as in the definition of the HBEP. Then $p(y)=\left(p_{1}(y), p_{2}(y)\right), y \in Y$, where $p_{1}$ and $p_{2}$ are real valued functions. Since $p$ is positive homogeneous $p_{1}$ and $p_{2}$ are positive homogeneous. Further by the subadditivity of $p$ and the ordering in $V,-p_{2}\left(y_{1}+y_{2}\right)+p_{2}\left(y_{1}\right)+p_{2}\left(y_{2}\right) \geqq 0$. That is, $p_{2}$ is positive homogeneous and subadditive. The function $p_{1}$, besides being positive homogeneous, satisfies only the following restriction: $p_{1}$ is additive whenever $p_{2}$ is.

Similarly, if $f(x)=\left(f_{1}(x), f_{2}(x)\right)$, then $f_{1}$ and $f_{2}$ are distributive and $f_{2}(x)$ $\leqq p_{2}(x)$, and $f_{1}(x)=p_{1}(x)$ whenever $f_{2}(x)=p_{2}(x)$. Thus, since the real number field $R$ has the HBEP, there exists a distributive extension of $f_{2}, F_{2}: Y \rightarrow R$, such that $F_{2}(y) \leqq p_{2}(y)$. Let $Z$ be the set of elements in $Y$ such that $F_{2}(z)$ $=p_{2}(z), z \in Z$. The set $Z$ is a cone, i.e., $z_{1}+z_{2} \in Z$, and $t z_{1} \in Z$, for $z_{1}, z_{2} \in Z$, $t \geqq 0$. For

$$
F_{2}\left(z_{1}+z_{2}\right) \leqq p_{2}\left(z_{1}+z_{2}\right) \leqq p_{2}\left(z_{1}\right)+p_{2}\left(z_{2}\right)=F_{2}\left(z_{1}\right)+F_{2}\left(z_{2}\right)=F_{2}\left(z_{1}+z_{2}\right),
$$

therefore, $F_{2}\left(z_{1}+z_{2}\right)=p_{2}\left(z_{1}+z_{2}\right)=p_{2}\left(z_{1}\right)+p_{2}\left(z_{2}\right)$.

As mentioned above, $p_{1}$ is additive whenever $p_{2}$ is additive. Hence $p_{1}$ is additive on $Z$. Thus, define $F_{1}^{\prime}(z)=p_{1}(z)$, for $z \in Z$, and extend it linearly to $L(Z, X)$, the space spanned by $Z$ and $X$, so as to be an extension of $f_{1}$. Now extend $F_{1}^{\prime}$ in whatever fashion to a distributive function $F_{1}: Y \rightarrow R$. Then the function $F(y)=\left(F_{1}(y), F_{2}(y)\right), y \in Y$, is a Hahn-Banach extension of $f$. Hence $V$ has the HBEP.

\section{BIBLIOGRAPHY}

1. S. Banach, Théorie des opérations linéaires, Warsaw, 1932.

2. M. M. Day, Notes on ordered linear spaces, University of Illinois, 1950, unpublished.

3. M. G. KreY̌n and R. A. Rutman, Linear operators leaving invariant a cone in a Banach space, [Uspehi Matematičeskih Nauk (N.S.) vol. 3 (23) (1948) pp. 3-95] Amer. Math. Soc. Translations, no. 26, 1950. 411-424.

4. R. J. Silverman, Invariant linear functions, Trans. Amer. Math. Soc. vol. 81 (1956) pp.

ILLINOIS INSTITUTE OF TECHNOLOGY, Chicago, Ill. 